\title{
Review \\ Fibrosis in connective tissue disease: the role of the myofibroblast and fibroblast-epithelial cell interactions
}

\author{
Thomas Krieg ${ }^{1}$, David Abraham ${ }^{2}$ and Robert Lafyatis ${ }^{3}$
}

\author{
1Department of Dermatology, University of Köln, Kerpener Strasse, D-50924 Köln, Germany \\ ${ }^{2}$ Department of Medicine, Centre for Rheumatology and Connective Tissue Diseases, Royal Free and University College, Rowland Hill Street, London, \\ NW3 2PF, UK \\ ${ }^{3}$ Rheumatology Department, Boston University of Medicine, Albany Street, Boston, Massachusetts 02118-2394, USA
}

Corresponding author: Thomas Krieg, thomas.krieg@uni-koeln.de

Published: 15 August 2007

This article is online at http://arthritis-research.com/content/9/S2/S4

(c) 2007 BioMed Central Ltd

Arthritis Research \& Therapy 2007, 9(Suppl 2):S4 (doi:10.1186/ar2188)

\begin{abstract}
Fibrosis, characterized by excessive extracellular matrix accumulation, is a common feature of many connective tissue diseases, notably scleroderma (systemic sclerosis). Experimental studies suggest that a complex network of intercellular interactions involving endothelial cells, epithelial cells, fibroblasts and immune cells, using an array of molecular mediators, drives the pathogenic events that lead to fibrosis. Transforming growth factor- $\beta$ and endothelin-1, which are part of a cytokine hierarchy with connective tissue growth factor, are key mediators of fibrogenesis and are primarily responsible for the differentiation of fibroblasts toward a myofibroblast phenotype. The tight skin mouse (Tsk-1) model of cutaneous fibrosis suggests that numerous other genes may also be important.
\end{abstract}

\section{Introduction}

Fibrosis, the result of excess synthesis and deposition of collagen, is a feature of many connective tissue diseases (CTDs) and is the hallmark of scleroderma (systemic sclerosis [SSc]). In this rare, progressive and life-threatening autoimmune disease, fibrosis affects not only the skin but also internal organs such as lungs and kidneys, leading to organ dysfunction and failure [1]. In SSc, fibrosis is the final stage in a series of pathological events that begins with vascular dysfunction, manifesting as altered vascular tone, endothelial activation and oxidative stress, followed by immunological activation and leucocyte-mediated extravascular inflammation [2]. A complex network of intercellular interactions that involves a diverse range of molecules, including growth factors, cytokines, chemokines and endothelin, is believed to drive the pathological events that ultimately result in uncontrolled connective tissue fibrosis. In this review, which describes results from in vitro systems and animal models of cutaneous and visceral fibrosis, we explore cellular and molecular events associated with the initiation and maintenance of fibrosis in CTDs.

\section{Initiators of fibrosis in connective tissue diseases}

Fibrosis arises from excessive collagen synthesis by fibroblasts [3]. Of the many potential mediators of fibrosis in CTDs, three molecular entities appear to be of particular importance: transforming growth factor (TGF)- $\beta$, endothelin (ET)-1 and connective tissue growth factor (CTGF/CCN2). In connective tissues, endothelin participates in stimulating the formation of myofibroblasts (the myofibroblast is a specialized type of fibroblast that is a normal cellular constituent of healing tissues) and increases levels of CTGF and matrix proteins [4] (Figure 1). It also interacts with other profibrotic cytokines including TGF- $\beta$, which is a key component in the induction and progression of fibrosis [5] and is a potent stimulator of CTGF. Indeed, CTGF is a common target for both TGF- $\beta$ and ET-1.

Data from experimental animal studies have shed light on the relationship between these three key mediators of fibrosis and pointed to the existence of a cytokine hierarchy. This hierarchy involves induction of ET- 1 by TGF- $\beta$, induction of CTGF by both ET- 1 and TGF- $\beta$, and potential mediation of the effects of TGF- $\beta$ and ET-1 on extracellular matrix (ECM) by CTGF [6] (Figure 2).

Evidence that TGF- $\beta$ increases ET-1 mRNA expression has come from several studies including an RNase protection assay, in which bovine endothelial cells were incubated in the presence or absence TGF- $\beta$ and ET-1 mRNA expression was measured over time. In cells exposed to TGF- $\beta$ there was a

CTD = connective tissue disease; CTGF = connective tissue growth factor; ECM = extracellular matrix; ET = endothelin; MAGP = microfibrilassociated glycoprotein; SMA = smooth muscle actin; SSc = systemic sclerosis; TGF = transforming growth factor; Tsk = tight skin. 


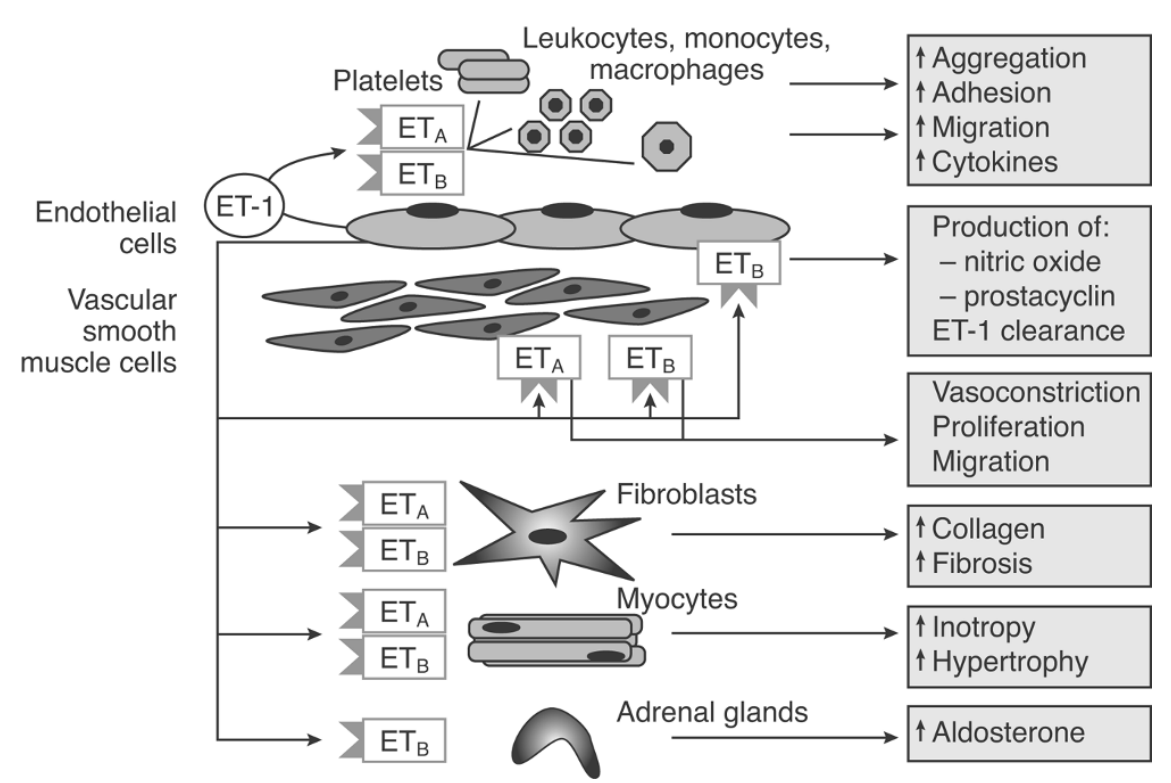

Effects of endothelin include stimulating myofibroblast formation, leading to a concomitant increase in collagen production and fibrosis. Binding of endothelin (ET)-1 to ET-1 receptor subtype $A\left(E T_{A}\right)$ and $E T_{B}$ has different effects in different cell types. The binding of ET-1 to smooth muscle cell $E T_{A}$ and $E T_{B}$ receptors leads to vasoconstriction and mitogenesis, and activation of $E T_{B}$ receptors on endothelial cells promotes the release of nitric oxide and prostacyclin, and plays a minor role in endothelial dependent vasodilatation. In fibroblasts ET-1 results in the increased production of collagen and leads to fibrosis. Reproduced with permission from Galiè et al. Cardiovascular Research (c) Elsevier 2004 [4].

\section{Figure 2}

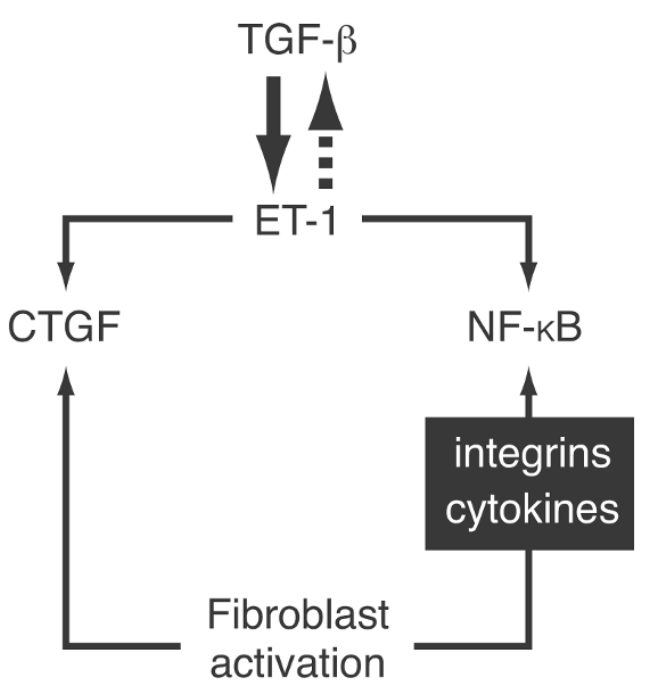

Schematic diagram of the hierarchy and interplay between ET-1, TGF- $\beta$ and CTGF. CTGF, connective tissue growth factor; $\mathrm{ET}$, endothelin; NF- $\mathrm{KB}$, nuclear factor- $\mathrm{KB}$; TGF, transforming growth factor. Reproduced from [6] by permission of the publisher (Taylor \& Francis Ltd, http://www.informaworld.com).

marked, dose-dependent increase in ET-1 mRNA expression, peaking at 4 hours after exposure [7]. Moreover, recent studies have also shown that TGF- $\beta$ can induce ET-1 production by pulmonary fibroblasts via a Smad-independent signalling pathway involving c-Jun amino-terminal kinase and the transcription factor activator protein-1. This pathway was found to be constitutively activated in injured cells [8]. Results from in vivo studies suggest that the effect of TGF- $\beta$ on ET-1 production occurs primarily in response to cell injury. In injured cells taken from bile duct ligated animals and exposed to TGF- $\beta$, there was a notable increase in ET-1 production in the injured stellate cells but not in injured endothelial cells (Figure 3a) [9].

These findings suggest that induction of ET- 1 by TGF- $\beta$ requires an activation step, perhaps dependent on inflammatory status or other cellular signals. Interestingly, blocking the effects of TGF- $\beta$ with a soluble TGF- $\beta$ receptor (STR) antagonist leads to inhibition of ET-1 production by both injured stellate and normal endothelial cells in vivo (Figure 3b) [9]. The concept of a cytokine hierarchy, in which ET-1 is induced by TGF- $\beta$, is also supported by data from experimental studies that showed that ET-1 does not increase TGF- $\beta$ gene expression or increase TGF- $\beta$ protein production in vascular smooth muscle cells [10].

As mentioned above, CTGF is a common target for both TGF- $\beta$ and ET-1. Recent studies showed that ET-1 regulates CTGF independently of TGF- $\beta$ and that, in turn, CTGF mediates ET-1 induced ECM accumulation. In an experimental study in which vascular smooth muscle cells were treated with 8 to $10 \mathrm{~mol} / \mathrm{l} \mathrm{ET}-1$, there was a marked increase 
Figure 3

(a)
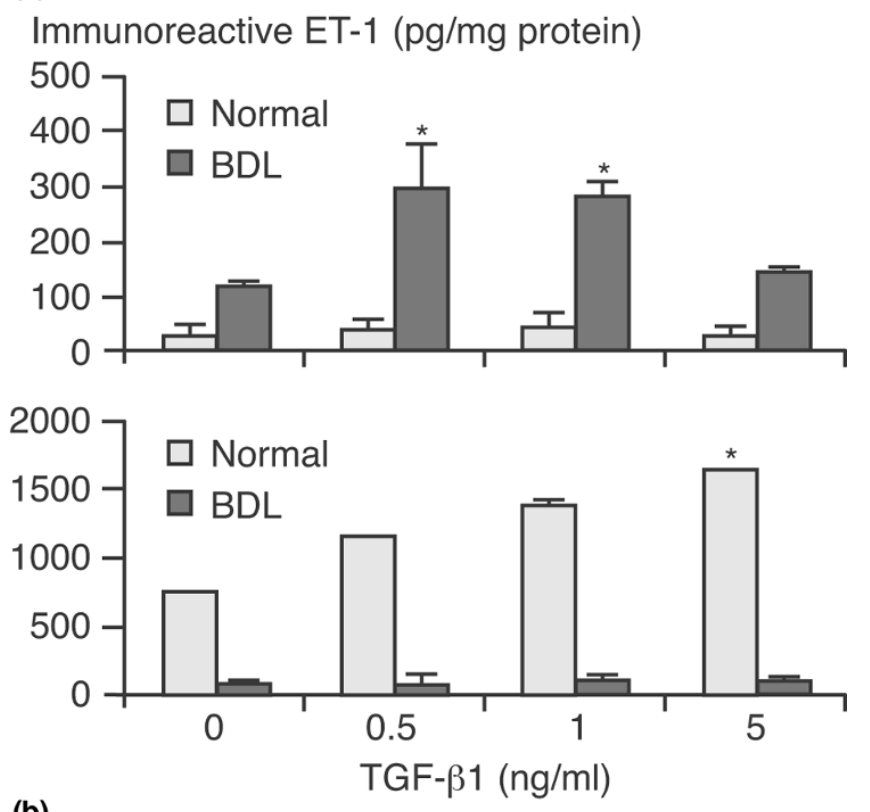

(b)

Immunoreactive ET-1 (pg/mg protein)

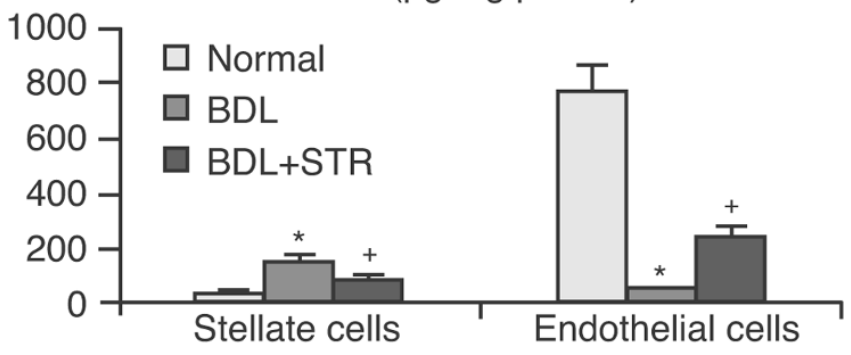

TGF- $\beta$ plays a pivotal role in the induction of ET-1. (a) Stellate (top panel) and sinusoidal endothelial (bottom panel) cells were isolated from normal rat livers or those injured by bile duct ligation (BDL). Transforming growth factor (TGF)- $\beta_{1}$ was applied at the indicated concentration in serum-free medium for 24 hours. Endothelin (ET)-1 was measured by radioimmunoassay. ${ }^{*} P<0.05$ versus no TGF- $\beta_{1}(n=5)$. (b) Stellate and endothelial cells were isolated from normal rat livers, and those after BDL and those after soluble TGF- $\beta_{1}$ receptor (STR) antagonist during BDL (BDL $+\mathrm{STR}) .{ }^{*} P<0.05$ versus normal and ${ }^{*} P<0.05$ versus $\mathrm{BDL}$ $(n=8)$. Reproduced with permission from Shao et al. Mol Biol Cell 2003 (c) The American Society for Cell Biology [9].

in type 1 collagen expression compared with control cells. However, when the treated cells were exposed to a CTGF antisense oligonucleotide that blocks the action of CTGF, type 1 collagen expression was markedly suppressed. Similar results were reported for levels of fibronectin, the production of which is also influenced by both ET-1 and CTGF [10].

From the available evidence it seems clear that TGF- $\beta$ can induce ET-1, although its induction is variable and may be dependent on other signals such as inflammation. Both TGF- $\beta$ and ET- 1 can induce CTGF, with ET-1 acting indepen- dently of TGF- $\beta$. CTGF, a cytokine that stimulates fibroblast growth and upregulation of collagen and fibronectin in vitro [11], is a difficult molecule to study experimentally, but there is evidence to suggest it mediates certain effects of TGF- $\beta$ and ET-1 on ECM production [12,13]. More complex interactions between TGF- $\beta$ and ET-1 are likely to depend on cross-regulation of receptors and activators.

\section{Models of the profibrotic microenvironment Models of organ fibrosis}

The in vivo effects of ET-1 on cardiac, renal and pulmonary tissue have been studied in a number of animal models of fibrosis. Experimental work on salt-induced hypertension in rodents (deoxycorticosterone acetate-salt hypertensive rats), for example, found a marked increase in fibrosis in the myocardium of affected animals compared with controls. However, when animals were given treatment with a selective endothelin-1 receptor subtype A antagonist, effective endothelin blockade resulted in no collagen deposition in cardiac tissue [14]. Earlier work on ET-1 transgenic mice, created by the transfer of the human ET-1 gene locus into the germline of mice, resulted in a pathological phenotype characterized by glomerulosclerosis, interstitial fibrosis and renal cysts without hypertension. In this model, pronounced renal fibrosis was associated with an age-dependent decrease in glomerular filtration that culminated in fatal kidney disease [15]. The severe ET-1-induced renal disease seen in this model of fibrosis is consistent with evidence from patients that an activated renal endothelin system is associated with glomerular and interstitial injury [15]. Other work in a transgenic mouse model has shown ET-1 over-expression to lead to the recruitment of inflammatory cells in respiratory tissues and subsequent development of progressive pulmonary fibrosis [16].

\section{Models of skin fibrosis}

More recently, a tight-skin mouse (Tsk-1) model has been used to enhance understanding of the underlying pathology of skin fibrosis in SSc. Tsk-1 mice develop tightening of the skin to the underlying tissues, due to a mutation in the gene encoding fibrillin 1. Fibrillin is a large ECM structural protein and the major component of microfibrils, found with or without associated elastin [17]. The primary role of fibrillin is to form the scaffolding for elastic fibres. In Tsk-1 mice, fibrillin appears to alter fibrillin matrix structure, with a notable increase in fibrillin in the superficial fascia of these animals. Tsk-1 mice exhibit increased elastogenesis in the superficial fascia (gain-of-function) and a loss of the dense elastic fibre band normally found at the interface between the intradermal muscle and deep connective tissue (loss-of-function).

To test the hypothesis that Tsk fibrillin causes skin tethering and fibrosis by altering interactions between fibrillin- 1 and other proteins, we conducted a series of experiments focusing in particular on microfibril-associated glycoprotein (MAGP)-1 and MAGP-2, fibulins, versican, decorin (small 
(a)
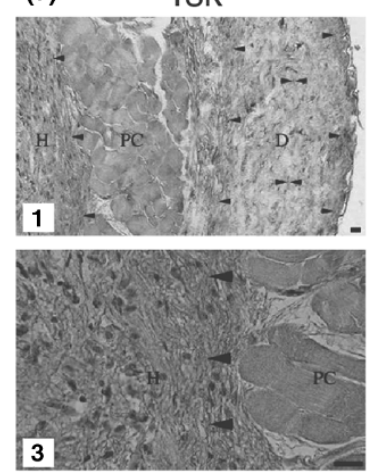

(b)

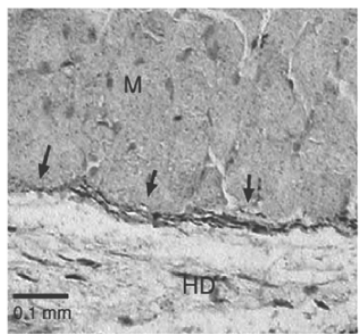

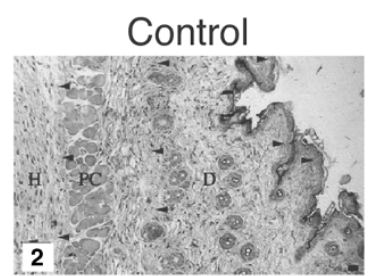
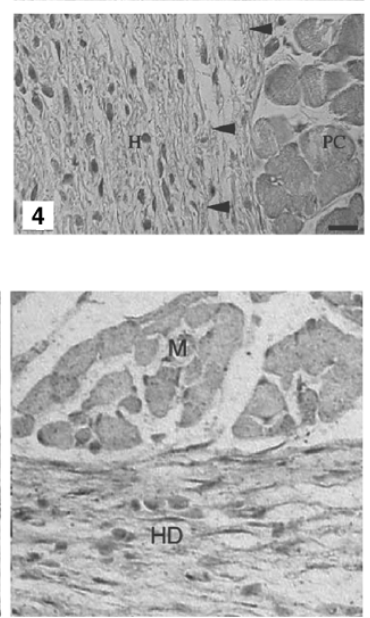

(c)
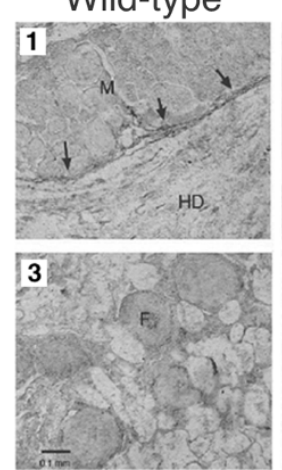

\section{Tsk}
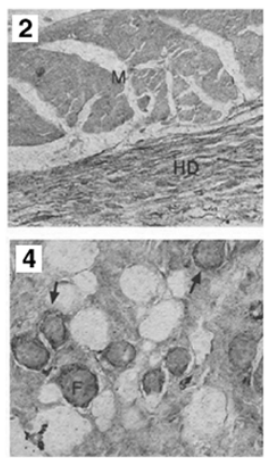

The Tsk-1 model of cutaneous fibrosis exhibits increased MAGP-2, loss of fibulin-5 and increased fibulin-2 matrix. (a) Tissue sections from tight skin (Tsk) mice (panels 1 and 3) and control (panels 2 and 4) mice were immunostained for microfibril-associated glycoprotein (MAGP)-2. MAGP-2 is indicated by arrowheads. MAGP-2 was detected at higher levels in all dermal layers (panel 1 versus panel 2). Hypodermis $(\mathrm{H})$ is shown (panel 3 versus panel 4). PC, panniculus carnosus; D, dermis. (b) Control (left panel) and Tsk (right panel) mice skin sections from 6-week-old mice were stained in parallel for fibulin-5 by immunohistochemistry. Positive staining is indicated by arrows at the muscle-connective tissue (M-CT) interface. Hypodermal connective tissue (HD) and hypodermal muscle (M) are indicated. Original magnification: 400x. (c) Control (panels 1 and 3 ) and Tsk (panels 2 and 4) mouse skin sections from 6-week-old mice were stained in parallel for fibulin-2 by immunohistochemistry. Positive staining is indicated by arrows (M-CT interface) and in panel 4 around hair follicles (F). (a) Reproduced with permission from Lemaire et al. Arthritis Rheum 2004 c John Wiley \& Sons/American College of Rheumatology [18]. (b) and (c) Reproduced with permission from [19].

leucine-rich repeat proteoglycans) and latent TGF- $\beta$ binding proteins, all of which are proteins known to interact or colocalize with fibrillin. Our results showed that MAGP-2 is upregulated in Tsk-1 skin and in cells expressing Tsk fibrillin, with the greatest increase seen in the hypodermis/superficial fascia of Tsk skin (Figure 4a) [18]. Tsk-1 mice also lose fibulin-5 expression at the interface between muscle and superficial fascia; fibulin-5 is important in regulating elastogenesis (Figure 4b). Fibulin-2, a molecule related to fibulin-5, was found to bind to both fibrillin and tropoelastin, and to be present in increased amounts in the superficial fascia (Figure 4c) [19]. Overall, these findings suggest that Tsk fibrillin alters the binding of fibrillin to other matrix proteins and that this altered binding appears to stimulate increased MAGP-2 and fibulin-2 matrix. Increased MAGP-2 or fibulin-2 may in turn, directly or indirectly, stimulate collagen and elastic fibre deposition leading to skin tethering. However, MAGP-2 and fibulin-2 mRNA is also upregulated in Tsk skin, raising the question of how matrix regulates matrix at the mRNA level. There is evidence that Tsk fibrillin-1 alters TGF- $\beta$ activity in Tsk skin as well as altering the binding of fibrillin to latent TGF- $\beta$ binding proteins. Interestingly, TGF- $\beta$ deleted heterozygotes exhibit less fibrosis than do littermate Tsk mice. Furthermore, studies using a hammerhead ribozyme to specifically silence the mutant fibrillin in skin fibroblasts derived from Tsk dermal biopsies revealed a striking decrease in the expression of TGF- $\beta$ dependent genes, including that encoding CTGF, in Tsk skin cells where the mutant fibrillin-1 had been deleted [20]. Ongoing studies in which global changes in gene expression in Tsk-1 mice are being assessed show that a variety of other proteins, in addition to those mentioned above, may be of importance in this model of skin fibrosis.

The Tsk-1 mouse clearly provides a good model for evaluating how matrix regulates its own composition. Although there is evidence from this model to suggest that Tsk fibrillin regulates gene expression through altered interactions with other matrix proteins, the identities of these mediators remain to be elucidated.

A further model is the bleomycin-induced model of dermal fibrosis, in which bleomycin has been found to increase expression of ECM proteins, potentially via the mediation of 
TGF- $\beta$ and CTGF/CCN2 [21]. This model has been widely used to evaluate potential antifibrotic drugs. For example, using this model, the protein kinase inhibitor imatinib mesylate (a selective, dual inhibitor of TGF- $\beta$ and platelet-derived growth factor) was shown to have potent antifibrotic effects [22], although perhaps not in later stages of fibrosis [23]. Peptide inhibitors of TGF- $\beta_{1}$ applied topically are also showing some promise [24]. Other agents targeting other components of the fibrotic process have similarly demonstrated attenuation of bleomycin-induced skin sclerosis in mice [25].

\section{Fibroblast to myofibroblast differentiation}

In comparison with normal skin, biopsies of skin from SSc patients show a significant increase in myofibroblasts, particularly in the deep dermis. These cells, which are characterized by the presence of stress fibres containing $\alpha$ smooth muscle actin (SMA), play a pivotal role in ECM deposition and wound contraction during the normal wound healing process [26]. A strong correlation has also been observed between myofibroblast intensity in the skin of SSc patients and the Rodnan skin score, which reflects disease throughout the body. Thus, cutaneous changes in SSc patients may reflect what is happening systemically, even though the disease may not be clinically evident in large parts of the body.

There has been much speculation on the origin of activated fibroblasts in the skin of SSc patients and what influences their differentiation toward a myofibroblast phenotype. There is evidence that activated fibroblasts may arise from local conversion of dermal fibroblasts by soluble factors, a finding that is consistent with our CTGF staining data. It is also possible that activated fibroblasts are recruited from circulating or resting mesenchymal precursor cells in the tissue, or induced by cell-cell contacts, cytokines or other chemokines, or selected as an active subpopulation by one or other mechanism. Alternatively, they may be generated by clonal selection. As is discussed in greater detail below, in wound healing the differentiation of fibroblasts toward a myofibroblast phenotype is governed by several factors, including the proximity of fibroblasts to keratinocytes, the presence of endogenous TGF- $\beta$, and direct cell-cell contact between fibroblasts and keratinocytes to allow initial TGF- $\beta$ activation.

\section{Orchestrating fibrosis: interactions between endothelial and other cells}

In the initial stages of any fibrotic condition, and certainly in SSc, there appears to be significant crosstalk between different cell types, including endothelial and mesenchymal cells. In vitro studies carried out during different stages of wound healing have shed light on the general activation mechanisms that are involved in fibrosis and on the intercellular crosstalk that influences cell behaviour. In the very early stages of wound healing, fibroblast activation is induced by epithelial cells in response to injury. In the skin, regulation during this very early phase of wound healing is due to direct contact between keratinocytes and the underlying fibroblasts, or to the release of cytokines from one cell to another, or, indeed, a combination of both. Interaction of fibroblasts and keratinocytes has also been found to modulate levels of two key enzymes that are involved in wound healing and remodelling: matrix metalloproteinase-2 and -9 [27]. This interaction may be critical for optimal healing quality at a later stage in the wound healing process.

There is, in addition, close interplay and crosstalk between the cells that control activation and downregulation of both cell types. Thus, fibroblasts are known to release keratinocyte growth factor, which induces keratinocytes to proliferate. Importantly, it also acts as a switch, turning on the production of other growth factors such as vascular endothelial growth factor, which then signal to endothelial cells $[28,29]$. In a recent series of experiments that included the incubation of normal keratinocytes with fibroblasts in vitro, it was shown that keratinocytes, either directly or indirectly, induced $\alpha$-SMA expression in fibroblasts [30]. Subsequently, it was discovered that induction of $\alpha$-SMA was associated with induction of collagen RNA; myofibroblasts induced by keratinocytes were found to produce large amounts of collagen. For induction to take place, however, the keratinocytes and fibroblasts had to be in very close contact. The genes induced as a result of the interaction between keratinocytes and fibroblasts included ECM genes such as collagens, tenascin $\mathrm{C}$, decorin, transglutaminase and lysylhydroxylase; cytoskeleton genes such as $\alpha-S M A$; and cellcell signalling genes such as monocyte chemoattractant protein 1, CTGF, activin A, ET-1 and angiopoietin (Table 1). Angiopoietin-related growth factor can potently promote adhesion, spreading and migration of keratinocytes, fibroblasts and endothelial cells, with the adhesion and migration events likely to be mediated by $\alpha_{v}$ containing integrins [31]. It has been suggested that $\alpha_{2} \beta_{1}$ integrins are responsible for cell attachment, spreading and migration. However, a recent study [32] showed that although the $\alpha_{2} \beta_{1}$ integrin is absolutely required for keratinocyte adhesion to collagens, for fibroblasts other collagen-binding integrins may partially make up for the lack of $\alpha_{2} \beta_{1}$ in simple adhesion to collagen monomers.

As later experiments have demonstrated, TGF- $\beta$ is responsible, at least in part, for the induction of $\alpha$-SMA in fibroblasts. Other important molecules involved in the regulation of myofibroblast differentiation included the cytokine granulocyte macrophage-colony stimulating factor, which inhibited myofibroblast differentiation via induction of the nuclear factor-KB pathway [33], and ET-1. In common with TGF- $\beta$, ET- 1 was also shown to induce $\alpha$-SMA. However, it appears to act synergistically with TGF- $\beta$ to induce the myofibroblast phenotype rather than acting alone (Figure 5). Although there is evidence that additional factors are 
Table 1

Selected genes induced by keratinocyte-fibroblast interactions

\begin{tabular}{ll}
\hline Type of gene & Details \\
\hline Extracellular matrix (ECM) & Collagens \\
& Hyaluronan synthase 2 \\
& Lysylhydroxylase 2 \\
& Transglutaminase 2 \\
& Tenascin C \\
& Decorin \\
& Syndecan 2 \\
& Monocyte chemoattractant protein \\
& (MCP)-1 \\
& Activin A \\
& Latent TGF- $\beta$-binding protein (LTBP)1 \\
Interleukin (IL)-6 \\
Connective tissue growth factor \\
(CTGF) \\
Heparin-binding epithelial growth \\
factor (HB-EGF) \\
Endothelin (ET)-1 \\
Angiopoietin 1 \\
Cyclo-oxygenase (COX)-2 \\
Granulocyte-colony stimulating factor \\
(G-CSF) \\
$\alpha$-Smooth muscle actin ( $\alpha$-SMA) \\
Integrins \\
Tropomyosin \\
Vasodilator-stimulated \\
phosphoprotein (VASP) \\
Myosin light chain kinase (MLCK) \\
\end{tabular}

Taken from [30].

involved, the data suggest that ET-1 and TGF- $\beta$ are responsible for most of the induction of $\alpha$-SMA-positive cells. In cell culture experiments in which an anti-TGF- $\beta$ antibody and inhibitor of ET-1 were added to the co-culture, there was significant downregulation of $\alpha$-SMA activity. Moreover, a recent study has shown TGF- $\beta$ preferentially induces ET-1 expression [34].

Results from these experimental studies indicate that highly complex epithelial-mesenchymal crosstalk is involved in controlling the extent to which fibroblastic cells are induced in the underlying mesenchyma and in their subsequent differentiation to myofibroblasts.

\section{Conclusion}

As the experimental studies described here illustrate, myofibroblasts play a central role in mediating tissue fibrosis, which is the major clinical manifestation of CTDs such as SSc. TGF- $\beta$, ET-1, and CTGF are among the most important molecular mediators of fibrogenesis, with TGF- $\beta$ and ET-1 intimately involved in the differentiation of fibroblasts toward a myofibroblast phenotype, characterized by the presence of stress fibres containing $\alpha$-SMA. Cell culture experiments show that direct cell-cell contact between fibroblasts and keratinocytes is important for TGF- $\beta$ activation and initiation of myofibroblast formation. Data from animal models of
Figure 5

aSMA expression (\%)

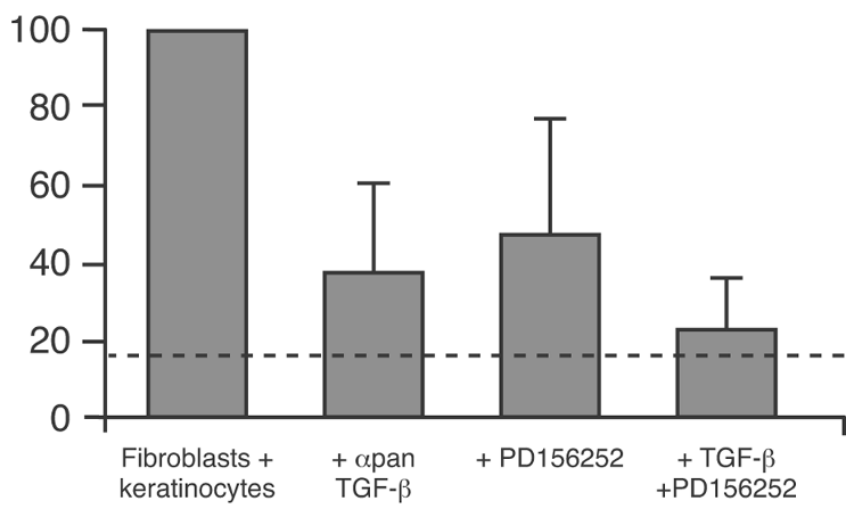

Synergistic effects of TGF- $\beta$ and ET- 1 in fibroblast activation. Signal intensities from Western blots of fibroblasts isolated from co-cultures of HaCaT keratinocytes and fibroblasts after 7 days in Dulbecco's modified Eagle medium $/ 0.5 \%$ foetal calf serum. $\alpha$-Smooth muscle actin ( $\alpha$-SMA) expression increased in co-cultures (see lane 1 ) as compared with fibroblasts alone (compare dotted line). Addition of an anti-transforming growth factor (TGF)- $\beta$ antibody or the endothelin (ET)-1 inhibitor PD 156252 resulted in reduced $\alpha$-SMA expression. Addition of anti-TGF- $\beta$ and PD 156252 blocked $\alpha$-SMA expression to almost basal levels [33].

cardiac, renal, pulmonary and cutaneous fibrosis confirm the importance of these profibrotic mediators in fibrogenesis, while also highlighting other molecular entities that may be important as well. The Tsk-1 model of cutaneous fibrosis, for example, illustrates the importance of Tsk fibrillin and how it regulates gene expression through altered interactions with other matrix proteins.

\section{Competing interests}

TK, DA and RL have received grant support and consulting fees from Actelion Pharmaceuticals Ltd, the manufacturer of bosentan.

\section{Acknowledgements}

The authors would like to acknowledge medical writing support funded by an educational grant from Actelion Pharmaceuticals Ltd.

This article is part of Arthritis Research \& Therapy Volume 9 Supplement 2: Advances in systemic sclerosis and related fibrotic and vascular conditions, and is based on presentations made at a symposium entitled Advances in systemic sclerosis and connective tissue disease, sponsored by Actelion Pharmaceuticals Ltd, held in Athens, Greece in April 2006. The full contents of the supplement are available online at http://arthritis-research.com/ supplements/9/S2. This supplement has been supported by an educational grant from Actelion Pharmaceuticals Ltd.

\section{References}

1. Denton CP, Black CM, Abraham DJ: Mechanisms and consequences of fibrosis in systemic sclerosis. Nat Clin Pract Rheumato/ 2006, 2:134-144.

2. Denton CP, Black CM: Scleroderma: clinical and pathological advances. Best Pract Res Clin Rheumatol 2004, 18:271-290. 
3. Mayes MD: Endothelin and endothelin receptor antagonists in systemic rheumatic disease. Arthritis Rheum 2003, 48:11901199.

4. Galiè N, Manes A, Branzi A: The endothelin system in pulmonary arterial hypertension. Cardiovasc Res 2004, 61:227-237.

5. Lasky JA, Brody AR: Interstitial fibrosis and growth factors. Environ Mealth Perspect 2000, 108:751-762.

6. Clozel M, Salloukh H: Role of endothelin in fibrosis and antifibrotic potential of bosentan. Ann Med 2005, 37:2-12.

7. Rodríguez-Pascual F, Redondo-Horcajo M, Lamas S: Functional cooperation between Smad proteins and activator protein-1 regulates transforming growth factor- $\beta$-mediated induction of endothelin-1 expression. Circ Res 2003, 92:1288-1295.

8. Shi-Wen X, Rodriguez-Pascual F, Lamas S, Holmes A, Howat S, Pearson JD, Dashwood MR, du Bois RM, Denton CP, Black CM, et al.: Constitutive ALK5-independent C-Jun N-terminal kinase activation contributes to endothelin-1 overexpression in pulmonary fibrosis: evidence of an autocrine endothelin loop operating through the endothelin A and $\mathrm{B}$ receptors. Mol Cell Biol 2006, 26:5518-5527.

9. Shao R, Shi Z, Gotwals PJ, Koteliansky VE, George J, Rockey DC: Cell and molecular regulation of endothelin-1 production during hepatic wound healing. Mol Biol Cell 2003, 14:2327-2341.

10. Rodriguez-Vita J, Ruiz-Ortega M, Rupérez M, Esteban V, SanchezLópez E, Plaza JJ, Egido J: Endothelin-1, via ET receptor and independently of transforming growth factor- $\beta$, increases the connective tissue growth factor in vascular smooth muscle cells. Circ Res 2005, 97:125-134.

11. Moussad EE, Brigstock DR: Connective tissue growth factor: what's in a name? Mol Genet Metab 2000, 71:276-292.

12. Gore-Hyer E, Shegogue D, Markiewicz M, Lo S, Hazen-Martin D, Greene EL, Grotendorst G, Trojanowska M: TGF-beta and CTGF have overlapping and distinct fibrogenic effects on human renal cells. Am J Physiol Renal Physiol 2002, 283:F707-F716.

13. Chujo $S$, Shirasaki $F$, Kawara $S$, Inagaki $Y$, Kinbara T, Inaoki M, Takigawa M, Takehara K: Connective tissue growth factor causes persistent proalpha2(I) collagen gene expression induced by transforming growth factor-beta in a mouse fibrosis model. J Cell Physiol 2005, 203:447-456.

14. Ammarguellat $F$, Larouche I, Schiffrin EL: Myocardial fibrosis in DOCA-salt hypertensive rats: effect of endothelin $\mathrm{ET}_{\mathrm{A}}$ receptor antagonism. Circulation 2001, 103:319-324.

15. Hocher B, Thone-Reineke C, Rohmeiss P, Schmager F, Slowinski T, Burst V, Siegmund F, Quertermous T, Bauer C, Neumayer $\mathrm{H}-\mathrm{H}$, et al.: Endothelin-1 trangenic mice develop glomerulosclerosis, interstitial fibrosis, and renal cysts but not hypertension. $J$ Clin Invest 1997, 99:1380-1389.

16. Hocher B, Schwarz A, Fagan KA, Thone-Reineke C, El-Hag K, Kusserow $\mathrm{H}$, Elitok $\mathrm{S}$, Bauer $\mathrm{C}$, Neumayer $\mathrm{HH}$, Rodman DM, et al.: Pulmonary fibrosis and chronic lung inflammation in ET-1 transgenic mice. Am J Respir Cell Mol Biol 2000, 23:19-26.

17. Siracusa LD, McGrath R, Ma Q, Moskow JJ, Manne J, Christner PJ, Buchberg AM, Jimenez SA: A tandem duplication within the fibrillin 1 gene is associated with the mouse tight skin mutation. Genome Res 1996, 6:300-313.

18. Lemaire R, Farina G, Kissin E, Shipley JM, Bona C, Korn JH, Lafyatis $\mathrm{R}$ : Mutant fibrillin 1 from tight skin mice increases extracellular matrix incorporation of microfibril-associated glycoprotein 2 and type I collagen. Arthritis Rheum 2004, 50:915-926.

19. Lemaire R, Korn JH, Schiemann WP, Lafyatis R: Fibulin-2 and fibulin-5 alterations in tsk mice associated with disorganized hypodermal elastic fibers and skin tethering. $J$ Invest Dermatol 2004, 123:1063-1069.

20. Menon RP, Menon MR, Shi-Wen X, Renzoni E, Bou-Gharios G, Black CM, Abraham DJ: Hammerhead ribozyme-mediated silencing of the mutant fibrillin-1 of tight skin mouse: insight into the functional role of mutant fibrillin-1. Exp Cell Res 2006, 312:1463-1474.

21. Yamamoto $T$, Eckes B, Krieg T: Bleomycin increases steadystate levels of type I collagen, fibronectin and decorin mRNAs in human skin fibroblasts. Arch Dermatol Res 2000, 292:556561.

22. Distler JH, Jungel A, Huber LC, Schulze-Horsel U, Zwerina J, Gay RE, Michel BA, Hauser T, Schett G, Gay S, et al:: Imatinib mesylate reduces production of extracellular matrix and prevents development of experimental dermal fibrosis. Arthritis Rheum 2007, 56:311-322.
23. Vittal R, Zhang H, Han MK, Moore BB, Horowitz JC, Thannickal VJ: Effects of the protein kinase inhibitor, imatinib mesylate, on epithelial/mesenchymal phenotypes: implications for treatment of fibrotic diseases. J Pharmacol Exp Ther 2007, 321:35-44.

24. Santiago B, Gutierrez-Canas I, Dotor J, Palao G, Lasarte JJ, Ruiz J, Prieto J, Borras-Cuesta F, Pablos JL: Topical application of a peptide inhibitor of transforming growth factor-beta1 ameliorates bleomycin-induced skin fibrosis. J Invest Dermatol 2005, 125:450-455.

25. Kimura $M$, Kawahito $Y$, Hamaguchi $M$, Nakamura $T$, Okamoto $M$, Matsumoto $Y$, Endo $\mathrm{H}$, Yamamoto $A$, Ishino $\mathrm{H}$, Wada $\mathrm{M}$, et al.: SKL-2841, a dual antagonist of MCP-1 and MIP-1 beta, prevents bleomycin-induced skin sclerosis in mice. Biomed Pharmacother 2007, 61:222-228.

26. Tomasek JJ, Gabbiani G, Hinz B, Chaponnier C, Brown RA: Myofibroblasts and mechano-regulation of connective tissue remodelling. Nat Rev Mol Cell Biol 2002, 3:349-363.

27. Sawicki G, Marcoux Y, Sarkhosh K, Tredget EE, Ghahary A: Interaction of keratinocytes and fibroblasts modulates the expression of matrix metalloproteinases-2 and -9 and their inhibitors. Mol Cell Biochem 2005, 269:209-216.

28. Werner S, Smola H: Paracrine regulation of keratinocyte proliferation and differentiation. Trends Cell Biol 2001, 11:143-146.

29. Werner S, Grose R: Regulation of wound healing by growth factors and cytokines. Physiol Rev 2003, 83:835-870.

30. Shephard P, Martin G, Smola-Hess S, Brunner G, Krieg T, Smola $\mathrm{H}$ : Myofibroblast differentiation is induced in keratinocytefibroblast co-cultures and is antagonistically regulated by endogenous transforming growth factor-beta and interleukin1. Am J Pathol 2004, 164:2055-2066.

31. Zhang Y, Hu X, Tian R, Wei W, Hu W, Chen X, Han W, Chen H, Gong $Y$ : Angiopoietin-related growth factor (AGF) supports adhesion, spreading, and migration of keratinocytes, fibroblasts, and endothelial cells through interaction with RGDbinding integrins. Biochem Biophys Res Commun 2006, 347: 100-108.

32. Zhang ZG, Bothe I, Hirche F, Zweers M, Gullberg D, Pfitzer G, Krieg $T$, Eckes $B$, Aumailley $M$ : Interactions of primary fibroblasts and keratinocytes with extracellular matrix proteins: contribution of alpha2beta1 integrin. J Cell Sci 2006, 119: 1886-1895.

33. Shephard P, Hinz B, Smola-Hess S, Meister JJ, Krieg T, Smola H: Dissecting the roles of endothelin, TGF-beta and GM-CSF on myofibroblast differentiation by keratinocytes. Thromb Haemost 2004, 92:262-274.

34. Castanares C, Redondo-Horcajo M, Magan-Marchal N, Lamas S, Rodriguez-Pascual F: Transforming growth factor-beta receptor requirements for the induction of the endothelin-1 gene. Exp Biol Med (Maywood) 2006, 231:700-703. 